\title{
L'hétérogénéité des expériences affectives et sexuelles de femmes vivant sans domicile fixe
}

\author{
Anne LAPORTE ${ }^{1}$, Erwan LE MÉNER ${ }^{2}$
}

\begin{abstract}
Résumé
A partir d'une trentaine d'entretiens semi-directifs avec des femmes résidant en centre d'hébergement d'urgence, nous avons mis en question, du point de vue de l'expérience de ces personnes, l'uniformité et le constat alarmant des descriptions ordinaires de la vie affective et sexuelle des femmes sans-abri. Leurs discours expriment la variété des expériences affectives et sexuelles et contredisent le déni, les clichés et le misérabilisme dont elles sont affublées. Si les conditions de vie difficiles tendent à contribuer, chez les femmes dont l'univers de sens est la rue, à un délaissement de soi, à l'adoption de conduites à risques, à une exposition accrue à des agressions, sexuelles notamment, à une réduction de la vie affective et sexuelle à une sphère de personnes proches, elles ne sauraient en aucun cas expliquer la diversité de l'expérience des femmes interrogées. Non seulement, des femmes qui se considèrent sans abri ont une sexualité désirée et connectent leur désir avec leur volonté de sortir de la rue, mais des femmes, qui vivent sans domicile, sans que la rue ne constitue leur univers de sens, se montrent également débrouillardes dans un environnement physique et relationnel diversement contraignant.
\end{abstract}

Vivre dans des conditions usantes, tant physiques que psychologiques, paraît de prime abord antinomique avec la construction de relations affectives et sexuelles, exigeant un souci de soi et des autres, du temps et de l'espace pour l'intimité.

Les travaux sociologiques sur les sans domicile sont nombreux et visent largement à documenter leurs modes de vie encore mal connus il y a peu de temps [1]. En dépit de l'inflation relative de ces travaux, la sexualité y occupe une place marginale, à l'instar des sans domicile dans les recherches en sciences sociales consacrées à la sexualité. De fait, rien ou presque n'est connu de leur vie affective et sexuelle et les rares pistes de lecture ignorent généralement le discours des premiers concernés.

Les femmes sans-abri sont ainsi l'objet de quelques descriptions dans la littérature sociologique, convergentes et alarmantes. Elles occupent, en amont de leur arrivée dans la rue, une position sociale extrêmement dominée, liée notamment à leur genre, et se meuvent, sans domicile, dans un environnement qui ne fait

\footnotetext{
Observatoire du Samusocial de Paris.

ISP-GAPP (Ens Cachan), observatoire du Samusocial de Paris.
}

que renforcer cette domination ${ }^{3}$. II est possible de parler d'une domination (subie) spécifiquement féminine, dans la mesure où l'exclusion sociale des femmes sans-abri tient en partie à leur statut de femme. Dans la rue, elles subissent des atteintes à leur féminité, répétées et durables, et doivent faire face à une domination masculine exacerbée $[4,5]$. À cet égard, leur vie affective et sexuelle semble à l'image de leur position sociale : dominée, davantage subie qu'élective, soumise au joug masculin [6]. La vie en couple de femmes sans domicile, la tenue de rapports sexuels n'exprimeraient qu'une demande de protection, vitale pour survivre dans des conditions extrêmes. L'attention, l'affection, voire l'amour déclarés, n'exprimeraient que l'illusion au principe du maintien des femmes dans leur position dominée. À partir du point de vue des femmes que nous avons rencontrées, seront mises en question l'uniformité et l'univocité de ces descriptions. Cette analyse est issue d'une étude satellite à l'enquête nationale sur le contexte de la sexualité en France (CSF) en $2006^{4}$, ayant pour objectif de rendre compte de la vie affective et sexuelle des sans domicile, en partant de l'expérience de certains d'entre eux.

\section{Méthode}

Trente entretiens semi-directifs ont été réalisés, vingt dans le cadre de la recherche sur la vie affective et sexuelle des sans domiciles fixes (SDF) et dix pour une étude sur la santé gynécologique de femmes de cette population ${ }^{5}$, tous selon la même grille entre avril 2003 et février 2005 auprès de femmes (âgées de 18 à 60 ans) résidant en centre d'hébergement d'urgence ou fréquentant des accueils de jour du Samusocial de Paris.

Les entretiens étaient structurés par des thèmes de recherche communs à ceux de l'enquête sur le contexte de la sexualité en France : biographie affective, puis sexuelle, avant et pendant la vie sans domicile; rapports sociaux de sexe, notamment parmi

\footnotetext{
Voir en particulier la contribution de J. Doherty, * Gendering Homelessness > in [2]. Le fait d'ētre femme semble néanmoins, toutes choses égales par ailleurs, diminuer les chances de devenir sans domicile [3].

Bajos N., Bozon M. « Le contexte de la sexualitè en France en 2005 -, Anrs, Réponse à l'appel d'offre du 15 septembre 2003. Bajos N, Bozon M, Eds. L'enquête sur la sexualité en France. Pratiques, genre et santé. Paris : La Découverte, 2008.

Cet article s'appuie sur deux rapports de l'Observatoire du Samusocial de Paris [7, 8]. Ces deux recherches ont èté soutenues respectivement par la Direction générale de la santé et par l'Institut national de prèvention et d'éducation pour la santè (Inpes).
} 
les SDF ; rapport au corps (hygiène, image de soi) ; rapport à la santé (santé ressentie, modes de recours aux soins, questions gynécologiques pour les femmes); relations familiales passées et présentes; conditions de vie actuelles (type d'hébergement, perception de sa situation générale, de son avenir proche) et rencontre imaginée.

Pour étudier la sexualité, nous sommes partis de la question: comment les personnes perçoivent et présentent-elles leur présence en centre d'hébergement ou d'accueil? La démarche est inductive, au sens où elle ne postule aucune définition de situation (WI Thomas) ${ }^{6}$ en surplomb des discours. La définition de la situation " être en centre " donnée par chaque interviewé, indiquait le partage d'une expérience caractéristique du sansabrisme. Nous faisions l'hypothèse que les traits de la sexualité variaient selon cette " définition de la situation ". Ensuite, pour former des catégories homogènes, nous avons utilisé le concept wébérien d'idéal-type, consistant en une activité de stylisation des principaux traits d'une catégorie ou situation historique, pour en faire ressortir les faits saillants [10].

Ainsi deux groupes se dégagent-ils d'après l'univers de signification qui, pour eux, donne sens à leur situation, et à partir duquel ils interprètent leur sexualité. Certains développent, comme prévu, une vision d'eux-mêmes et de leurs actions comme s'inscrivant quasi-exclusivement dans le cadre de la vie sans domicile fixe. Ces individus ont été répartis sur un premier axe selon la présence ou l'absence d'activités déployées pour sortir de la rue ou saisir des opportunités pouvant se présenter à eux. Un second axe les discrimine selon leurs stratégies face au stigmate ou à l'étiquette SDF (l'étiquette, acceptée ou dévoyée, est en tout cas admise : ces gens savent qu'on les considère comme SDF). Mais nombre d'individus ne situent pas leur discours dans l'univers de sens de la rue : soit ils se définissent par une appartenance à une catégorie distincte de celle de SDF, soit être en centre d'urgence ne prend sens qu'en rapport avec la situation passée. La rue peut alors être considérée comme une parenthèse biographique, comme une ressource pertinente pour rompre avec une situation passée dévalorisée mais prégnante, ou comme une situation ne posant pas problème, inscrite dans le prolongement d'une trajectoire biographique marquée par l'instabilité résidentielle.

\footnotetext{
6 WI Thomas, * Définir la situation *, extrait de The Unadjusted Girl, [1923], traduit dans Grafmeyer $\mathrm{Y}$, Joseph I. (dir.) [9]. Ce concept se rapporte à une réalité difficile à cerner par les questionnaires et la statistique : " la phase d'examen et de délibération qui précède toute conduite autodéterminée », I'homme, au contraire de l'animal, se caractérisant par une capacité à prendre des décisions réfléchies. Cette phase possède une double dimension, de définition du présent, mais également d'aiguillage de la conduite future. Comme le dit Thomas, * c'est toute une ligne de vie, toute une personnalité qui découlent peu à peu d'une série de telles définitions $*$. Néanmoins, Thomas précise immédiatement que l'individu ne peut établir de telles définitions sans interférer avec le reste de la société et les agents de socialisation, telles la famille et la communauté, * qui ont déjà défini tous les grands types de situation susceptibles de se présenter " : la définition de la situation, formulée subjectivement, n'est pas moins conditionnée par des instances sociales préexistantes. L'analyse de chaque entretien et de chaque définition de situation passe donc par l'articulation d'une perspective indigène sur le fait d'être dans la rue, et de contraintes exogènes pesant sur le point de vue indigène.
}

\section{La variété des expériences affectives et sexuelles des femmes sans-abri}

Nous verrons d'abord que les femmes qui se définissent comme sans-abri exercent une sexualité, dont l'importance, la visée et la signification se rapportent directement au stigmate dont elles se reconnaissent affligées (fatalistes, résignées). Soulignons immédiatement que toutes les femmes interrogées ne décrivent pas une telle attitude, y compris parmi celles qui se définissent comme sans-abri, dont certaines cherchent fermement à se défaire de l'étiquette de SDF et à sortir de la rue, et projettent leur vie affective et sexuelle dans cette direction (volontaires). Nous présenterons en détail un $\operatorname{cas}^{7}$ de chaque catégorie.

II faut encore ajouter, tel sera le cœur de notre seconde partie, que des femmes ne se considèrent pas comme sans-abri, et expérimentent leur vie affective et sexuelle d'après des perspectives étrangères à leur statut de SDF.

\section{La vie affective et sexuelle des femmes se considérant sans-abri}

\section{Les fatalistes}

Certaines femmes définissent leur présence en centre d'hébergement dans un univers de sens que nous disons "de la rue ", dans la mesure où chacune se reconnaît stigmatisée ${ }^{8}$ par le statut de SDF et présente la sortie de rue comme l'échappatoire à l'étiquette (cela n'implique pas que toutes les femmes ne cherchent pas à retourner le stigmate, ni, non plus, qu'elles envisagent toutes de sortir de la rue). Leur vie affective et sexuelle, comme tout autre domaine d'expérience, prend sens par rapport à cet univers de sens et aux définitions de situation qui y sont associées. Moins une femme s'efforce de retourner le stigmate, moins elle imagine sortir de la rue et agit en conséquence, plus elle se définit comme "fataliste", plus sa vie affective et sexuelle lui parait insatisfaisante mais stabilisée. L'insatisfaction s'applique aux partenaires, aux conditions d'exercice de l'intimité, sexuelle notamment, et aux pratiques elles-mêmes, sans aucune volonté de changement. Une incapacité à moduler des pratiques dépréciées s'observe, qui nous semble analogue à la non-perception de certains de leurs comportements comme des conduites à risque (ainsi que définies par la santé publique), en

\footnotetext{
Comme nous l'avons dit, les catégories dans lesquelles nous classons les entretiens stylisent des expériences. Dans ce mode d'ordonnancement des données, chaque entretien est mobilisable comme un cas illustrant les traits généraux de sa catégorie. Le choix de ne présenter qu'un entretien pour décrire une catégorie est rhétorique. Tel entretien de telle catégorie ne présente en soi pas plus d'intérêt que tel autre. Mais ne choisir de ne parler que d'un entretien (connu dans ses moindre détails par les auteurs) permet d'en parler davantage et mieux et, donc, nous l'espérons, de sensibiliser plus efficacement aux enjeux qu'il soulève, et qui valent pour les individus (interrogés ou non) de sa catégorie. Pour une démarche comparable, voir [11].

8 Nous faisons référence au travail d'E. Goffman [12]. * Le nom de stigmate servira donc à désigner un attribut qui jette un discrédit profond, mais il faut bien voir qu'en réalité c'est en termes de relations et non d'attributs qu'il convient de parler $*$ (p. 13).
} 
tant qu'elle s'enracine dans des routines qui stabilisent les personnes dans la rue.

Nöelle, 46 ans, vit sans domicile par intermittence depuis huit ans. Après son divorce, elle perd son travail, puis est expulsée de son appartement. Elle passe la majeure partie de son temps dans l'espace public, en compagnie de son petit-ami, Eric, rencontré six ans plus tôt. Noëlle ne s'étonne nullement de ne pas trouver de travail, elle a tout à fait conscience de sa dégradation physique et du stigmate qu'elle véhicule :

"On se laisse tomber, on se dévalorise, on se sent mal, on a tous les autres qui vous critiquent autour parce qu'on a les vêtements sales, vous sentez pas bon, à cause des vêtements. [...] Le plus dur, c'est les autres autour qui voient qu'on a un problème parce qu'on est avec des paquets, on a le linge sale, on est mal habillée ou bien on est tout le temps en train de tourner avec des paquets".

Ce fatalisme, perceptible dans ses activités quotidiennes, en fait une figure féminine du " clochard " [13], stade ultime du processus de disqualification, ou rupture sociale [14].

Toutefois, le délaissement de soi n'est pas concomitant d'un désinvestissement de la sphère affective. Noëlle se dit " très amoureuse " d'Eric, et exprime également un fort désir de maternage envers lui. Son propos, comme celui de nombreuses femmes (pas seulement fatalistes), contrarie l'idée selon laquelle les femmes SDF se mettent en couple uniquement pour bénéficier de la protection d'un homme. Dans notre échantillon, les femmes les plus fatalistes, toutes en couple, sont à la fois les plus exposées à toutes formes d'offenses dans l'espace public, bien conscientes de ces dangers, et celles qui déclarent défendre leurs partenaires qui, eux, ne semblent pas les protéger. Ainsi Noëlle a-t-elle peur des violences physiques ou sexuelles, auxquelles elle a du reste déjà été confrontée (elle relate un viol subi par elle et Eric dans une laverie automatique). Cette absence de soutien physique des hommes, exigeant des combines pour se protéger soi-même, ajouté à des conditions de vie stigmatisantes, et à l'impossibilité de tenir certains rôles sociaux féminins, dont celui de mère, fait dire à ces femmes qu'elles perdent leur féminité. Noëlle en témoigne :

"Je me suis habituée, mais c'est pas bien. C'est pas bien qu'une mère soit séparée de ses enfants au niveau psychologique. Je perds beaucoup de moi. Je perds aussi ma féminité comme ça et je perds de l'estime de moi. Je me reconnais plus [...]. J'ai l'impression que je ressemble de plus en plus à l'homme [...]. I C'est la vie à la rue? I Oui, la vie, elle est dure, le fait de ne plus rien avoir du tout, c'est insatisfaisant, de plus avoir de relations sexuelles, je me masculine et je suis tout le temps en train de porter des sacs et d'avoir des charges sur les épaules, des responsabilités, à me planquer. Je me masculine un peu des fois physiquement parce que je suis en danger dehors, pour la sécurité. J'ai peur des viols, des maltraitances. Je veux pas montrer trop mon corps parce que je suis presque tout le temps avec des hommes. Je suis un peu conditionnée. J'aime bien être avec des hommes et j'aimerais bien être un homme des fois, ça simplifierait ma vie."

Cette tendance à l'inversion des rôles sociaux de sexe, relevant des conditions de vie extrêmes des partenaires, modifie les apparences, et détériore le désir, de part et d'autre, souligne encore Noëlle :

"Ça, le fait d'être à la rue ça tue les désirs, ça émousse les plaisirs et ça met au point mort les désirs sexuels. C'est le point mort. C'est rien, c'est rien de le dire, je n'aime plus, je n'aime plus les relations sexuelles, je deviens foutiste complètement, sexuellement, c'est pour ça qu'il a envie d'aller ailleurs. [...] J'ai l'impression qu'il veut aller avec une autre parce qu'il m'aime plus et que je l'excite plus sexuellement, physiquement. II regarde les autres plus que moi. "

Nöelle, pourtant, considère que le partage charnel est capital pour un couple. Mais Noëlle ne présente pas moins la dégradation de leurs apparences et de leurs désirs, ainsi que les difficultés d'accéder à des conditions normales d'exercice de l'intimité, comme des obstacles rédhibitoires. Ne pas être vus comme un couple légitime, ne pas avoir de chambre à soi, laisse Noëlle penser qu'elle n'a pas "droit » à la sexualité :

" On est bloquée, on est refoulée, on est mal dans sa peau et on méprise les organes sexuels et on méprise le partenaire, on le voit changer de visage, on le voit changer aussi... On ne le regarde plus du tout, on cherche même plus à force parce qu'on est tout le temps, on a l'impression d'être frustrée, punie et qu'on n'a pas droit, on n'a pas droit, alors on n'y fait plus attention. "

Moins désirante d'Eric, Noëlle a " pris l'habitude de se satisfaire toute seule" : en dormant, "par l'esprit" ou sous la douche, en se masturbant. Le fatalisme de Noëlle s'exprime donc dans sa vie affective et sexuelle, et se traduit aussi par une conduite paradoxale vis-à-vis des risques d'infections sexuellement transmissible. Noëlle, dont Éric est le seul partenaire sexuel depuis plusieurs années, n'est pas sûre que ce soit réciproque. II s'éloigne d'elle, elle se demande s'il n'a pas des aventures, elle déplore son détachement de plus en plus marqué. Pas plus qu'elle n'essaie de changer d'apparence pour le reconquérir, elle ne cherche à se prémunir contre des risques d'infection sexuellement transmissible. Pourtant Noëlle a peur du sida, elle a vu "d'autres en mourir ". Mais elle n'utilise pas le préservatif car elle considère qu'il n'est pas fiable, il n'empêche ni la grossesse, ni la maladie, et " ça tue la moitié du plaisir ". Elle ajoute : " J'ai confiance. II me dit qu'il n'a personne d'autre que moi quand il est avec moi. Et moi je lui dis pareil ». Le repli affectif sur le couple est tout fait prégnant chez les femmes fatalistes: le couple est à la fois le miroir de ses propres vulnérabilités, de ses déceptions et de ses frustrations et le support principal du maintien de soi, le gage que l'on compte encore pour quelqu'un, quitte à ne pas voir les risques ou les dangers afférant, ou à ne pas vouloir ou pouvoir s'en détourner. 


\section{Les résignées}

Dessinant, à la différence des fatalistes, l'espoir de sortir des dispositifs d'urgence, bien qu'elle soit accablée par le stigmate, Bénédicte représente une figure des " résignés ». Depuis 14 ans, elle fait des allers et retours dans les centres d'hébergement au rythme des disputes conjugales et de la fatigue de la rue. Sa vie quotidienne, à l'instar de celle des fatalistes, s'organise au fil de routines satisfaisant des besoins élémentaires, routines à la fois aliénantes et structurantes.

Si toutes les résignées reconnaissent des obstacles objectifs à l'exercice de leur sexualité, elles pointent néanmoins des possibilités d'adaptation, liées visiblement à leur connaissance de la ville, du circuit d'assistance et de leurs niches écologiques. Une des contraintes les plus citées est sans conteste l'absence de lieu intime et sécurisant pour le couple, permettant de se retrouver à l'abri de regards indiscrets. Cette embûche n'implique pourtant pas l'absence de relations sexuelles, en témoigne Bénédicte :

" $Y$ en a beaucoup qu'arrivent pas à comprendre qu'on arrive quand même à avoir des relations sexuelles. C'est dur mais bon, on essaie de faire avec. "

Les résignées, à l'image de Bénédicte, paraissent ainsi plus enclines à se satisfaire des combines mises en œuvre pour exercer leur sexualité que les fatalistes, qui redoublent leur disqualification sociale par une disqualification des espaces dans lesquels ils peuvent se retrouver. Les résignés, plus que les fatalistes, davantage captifs de leur environnement familier, se montrent capables de se démarquer spatialement, comme socialement, des lieux et des personnes qui nuisent à leur vie privée. Écoutons à ce propos Bénédicte répondre à nos questions :

"Au niveau des relations de couple, est-ce que vous avez eu des relations affectives depuis que vous êtes SDF ? / Oui, mon mari [que j'ai rencontré à Nanterre], mais c'est dur quand même d'avoir une relation qui tienne en étant à la rue. / Justement, vous pouvez m'en parler? / Disons que c'est un peu difficile quand même parce que quand on est à la rue, disons qu'y a des trucs. Comme là, j'étais avec mon mari, on travaillait tous les deux [à Nanterre], mais bon y a souvent des gens qui sont jaloux : oh, je voulais sortir avec lui, tu me l'as pris. Des trucs comme ça. Bon, c'est quand même assez difficile à tenir. Alors bon, les gens sont assez jaloux quand même, des relations que les autres ils ont avec un homme."

Bénédicte, si elle parvient avec son compagnon à s'écarter des pressions des gens alentour et à s'aménager des espaces intimes, n'est pas moins prudente et se sait, comme femme, particulièrement vulnérable à toutes sortes d'" offenses territoriales " $[15$, p. 62-70]. Les élans de séduction dont elle est l'objet, lorsqu'elle vit sans son époux dans la rue, l'inquiètent toujours, lui apparaissant porteurs de menaces. Toutefois, elle se fait raccompagner par des amis jusqu'à la porte des centres d'hébergement (sans acheter cette sécurité en vendant son corps), et ne cherche pas une protection particulière auprès d'un homme. Si elle limite le nombre de ses partenaires, c'est par choix, parce qu'aucun ne lui plaît assez (mais elle ne cherche pas, au contraire des " volontaires » dont nous allons parler, à rencontrer l'homme qui la satisferait). Dans l'attente (passive) d'un grand amour qui lui ferait quitter durablement la rue, Bénédicte se montre d'autant plus prudente qu'elle ne pourrait qu'avec peine utiliser des préservatifs ; il semble, en creux de ses déclarations, que les relations sexuelles de Bénédicte aient lieu sans protection, reposant sur un principe de confiance en son partenaire (ce comportement se retrouve chez les résignées comme chez les fatalistes, mais chez les premières, la confiance accordée dans le partenaire est aussi une confiance en un avenir meilleur).

Les résignées attendent donc un grand amour, mais au quotidien, elles parviennent à assouvir leurs attentes sexuelles, et vivent entourées de proches. Résignées, elles ne mettent guère en œuvre de plan pour sortir de la rue, ou rencontrer cette personne désirée, et se montrent assez peu sensibles aux risques de santé qu'elles encourent dans la rue.

\section{Les volontaires}

Mais parmi les femmes dont l'univers de sens est la rue, certaines se différencient par le rapport distancié et distanciant qu'elles entretiennent au stigmate de sans-abri, ainsi que par leur perception de leur capacité à faire face, l'expression de la confiance qu'elles ont en elles et en l'avenir, la conviction qu'elles vont " s'en sortir». Nous les avons appelées les " volontaires".

Sylvie, 23 ans, tient à rentrer dans la norme, psychologiquement comme physiquement, cherchantà prouver auxautres, travailleurs sociaux ou pairs, qu'elle peut être considérée comme autonome et responsable et non plus comme une " handicapée ». Sylvie souffre, outre du stigmate attaché à son statut de sans-abri, de son obésité, qui l'empêcherait de vivre une sexualité épanouie. Pour changer, elle envisage de faire appel à la chirurgie esthétique. Son discours décrit de nombreuses compétences acquises à la rue, dont une connaissance avertie du dispositif d'assistance, mise à profit dans ses démarches. Sylvie mobilise également son expérience amoureuse, développée dans la rue, en vue de quitter cet univers, et celui de la psychiatrie. Elle cherche activement un partenaire qui ne soit " pas dans la galère ", avec qui elle pourrait nouer une relation stable. Car si Sylvie n'aime pas son corps, elle ne décrit pas moins son attrait aux yeux d'amants, qui posent sur elle un regard bien différent de celui, stigmatisant, qu'elle perçoit d'ordinaire. Sylvie multiplie les rencontres. Du reste, elle n'ignore pas la possibilité de maladies sexuellement transmissibles, semble bien informée des modes de transmission du VIH (faisant au demeurant partie d'une génération ayant débuté sa vie sexuelle dans le milieu des années 1990 et reçu une information sur le sida à l'école) :

"Voilà, dit-elle au sujet d'un de ses amants, je lui ai expliqué et le gars, il me dit : oui, le sida, ça s'attrape avec la salive. Je lui 
dis : non, je sais quand même, j'ai été à l'école et c'est l'école qui m'ont appris et je sais très bien que le sida ça s'attrape pas par la salive, ça s'attrape par le sang et par les rapports sexuels sans protection. "

Pourtant, elle ne se protège pas systématiquement. Elle est ainsi tombée involontairement enceinte. Mais cette prise de risque, que Sylvie désigne d'ailleurs du ressort de la responsabilité de son partenaire ( il a fait une connerie [en ne mettant pas de préservatif ") ne paraît pas explicable par l'environnement de la rue. Cette attitude se rapporte plus à un comportement général dans le rapport sexuel, fait de passivité et d'hésitation, qu'à un problème de ressource financière (acheter des préservatifs) ou écologique (ses partenaires ont généralement un logement).

En bref, il apparaît que la vie affective et sexuelle des femmes se considérant comme sans-abri les satisfait d'autant moins que celles-ci sont sédentarisées dans la rue. À l'opposé, plus les femmes travaillent à défaire " leur identité virtuelle » [12, p. 12] et à " sortir de la rue ", plus elles se montrent capables de construire des relations affectives qui se connectent avec leur " volonté de sortie ". La féminité peut alors être un atout distinctif pour nouer des contacts avec des personnes insérées. Les situations de ces femmes, fatalistes, résignées, ou volontaires ${ }^{9}$, n'épuisent pourtant pas la variété des situations et des expériences affectives et sexuelles rencontrées dans la rue.

\section{La vie affective et sexuelle de femmes dont l'univers de sens n'est pas la rue}

Nombre de femmes bénéficiaires du dispositif d'urgence sociale ne se définissent pas par rapport à un stigmate de sans-abri, et ne décrivent pas la sortie de rue comme le moyen de défaire ce stigmate. Ces femmes vivent sans logis mais leurs préoccupations se rapportent indirectement à leur présence dans la rue. Des veuves, des femmes cherchant à s'éloigner de leur époux, des jeunes femmes migrantes en rupture momentanée avec l'insertion, d'autres encore, confrontées, dans l'urgence, à une nouvelle épreuve et déterminées à surmonter cet obstacle comme un autre : autant de personnes dont la présence en centre d'urgence se définit en référence à des événements antérieurs à l'arrivée dans la rue (la perte de l'époux, la rupture conjugale etc.) La vie en centre est fonction de questions (bien finir son existence; reconstruire sa vie à distance de son ancien partenaire ; retourner chez soi etc.) qui lui préexistent. Ces questions organisent et structurent la vie de ces personnes, et notamment leurs expériences affectives et sexuelles. Ainsi pouvons-nous comprendre, par exemple, que des femmes dont l'activité sexuelle est éteinte n'associent aucunement cette suspension à leur présence en centre d'hébergement, ni n'affirment qu'elle nuise à leur fémi-

\footnotetext{
9 Les catégories dans lesquelles nous classons ces personnes ne doivent pas faire oublier que l'exclusion est affaire de processus tout autant que d'états. Comme la majorité de la recherche contemporaine, nous souscrivons à une définition processuelle et relationnelle de l'exclusion [16].
}

nité, et que des femmes modulent, de manière satisfaisante, leurs conduites affectives et sexuelles dans la rue

Une suspension de l'activité sexuelle, autonome de la vie dans la rue

Des femmes nous ont dit ne plus avoir ni désirer de relations sexuelles. Nous pouvions penser, a priori, que cette extinction du désir et de l'activité sexuelle s'expliquait par les difficultés de la survie, se nourrir, dormir, se protéger étant des besoins prioritaires, et la promiscuité des relations sociales, le manque d'intimité, la faible attirance pour des gens alentour dissuadant toute recherche ou saisie d'occasion amoureuse ou sexuelle. Difficultés que nous racontent bel et bien ces femmes. Marion, franco-camerounaise de 55 ans, depuis six mois dans la rue, à la suite de la vente de l'appartement qu'elle louait, raconte son inquiétude et ses difficultés pour subvenir à des besoins élémentaires, juste après son arrivée en centre d'hébergement :

"Au début, c'était difficile? / Très difficile, c'était ma première fois de ma vie, je ne connaissais pas comment s'y prendre, comment il faut faire, où étaient les toilettes, il fallait chercher, c'est dans le couloir."

Beaucoup, et pas seulement des femmes dont la sexualité est interrompue, parlent de leur dégoût d'emblée, pour les " clochards", dénoncent les "racontars", un environnement où la rumeur se propage à toute vitesse. Quand l'enquêteur demande à Marie, qui réside depuis quelques semaines en centre d'hébergement d'urgence, s'il lui arrive d'être attirée par des personnes vues dans des centres, elle répond avec ironie : "Jusque-là, je n'ai pas encore trouvé un prince charmant (rires). J'ai pas encore trouvé ! ", et ajoute, à propos des autres hébergés : "Ca risque plus de me dissuader ".

Mais ces femmes, qui n'ont plus d'activité sexuelle et qui décrient à maints égards leur environnement, disent aussi avoir appris à vivre dans les centres, à surmonter ce qui leur apparaissait d'abord comme des obstacles, pour pouvoir satisfaire pleinement certaines de leurs attentes, en matière de soin corporel notamment. Marion attache, par exemple, beaucoup d'importance à sa toilette, qui semble la ressourcer :

"Alors la douche, j'adore la douche, me laver trois fois par jour. Ah oui, le matin, brosser mes dents, me laver. A midi, avant le repas vers 11 heures, malheureusement ça me manque. Je fais que deux fois par jour : le matin me laver et je reviens le soir au foyer et là, avant de me coucher, je vais faire ma douche, brosser mes dents, mettre l'huile et me coucher et mon traitement. La propreté, j'adore, c'est mon dada. On se sent à l'aise, on dort comme un petit poussin ”

Ces femmes parviennent donc à garder une bonne image d'elles-mêmes. Et elles expliquent la suspension de leur désir ou de leur vie sexuelle par un événement antérieur à l'arrivée dans la rue (comme la perte de l'époux), un processus plus long de 
délitement du désir, lié à l'âge et à la durée de la relation amoureuse, ou le souci, obsédant, de d'abord surmonter l'obstacle qui se présente à elles (échapper aux griffes de l'époux, retourner chez soi, trouver un travail et un logement) ou tout simplement à l'absence de bonne rencontre. En tout cas, elles n'imputent pas l'interruption de leur activité sexuelle à leur situation de sansabri ; elles ne distinguent pas dans l'environnement matériel ou relationnel d'éléments suffisamment offensants ou contraignants pour suspendre leur désir.

Par conséquent, les femmes de notre échantillon, dont l'activité sexuelle est la plus en sommeil, en rendent raison en convoquant des événements décisifs ou des attentes non satisfaites, qui ne dépendent pas de leur présence dans la rue. Vivre dans la rue ne comporte pas moins des inconvénients, pour prendre soin de soi, ou pour maintenir des relations affectives, mais ces obstacles peuvent être contournés.

\section{Une sexualité aux significations inchangées}

De façon semblable, les femmes (ne se considérant pas comme sans-abri), qui exercent leur sexualité en vivant sans domicile, parviennent à surmonter d'éventuelles difficultés liées à leurs conditions de vie pour mettre en œuvre leur sexualité de façon satisfaisante (à l'instar des volontaires par exemple), mais le sens qu'elles accordent à leur vie sentimentale ne paraît pas transformé par leur statut de sans domicile.

Aude fait partie de ces femmes pour lesquelles la rue n'est qu'une discontinuité de plus dans une existence semée d'embûches. Aude (22 ans) a été placée dans des familles d'accueil de 5 ans à 18 ans. Son père, sénégalais, polygame, vit une partie du temps au Sénégal et sa mère est présentée comme toxicomane. Aude a été violée à l'âge de 13 ans, son agresseur est en prison. Elle a perdu plusieurs proches auxquels elle tenait, son frère, mort sous ses yeux, son grand-père, et d'autres encore. Elle a tenté de vivre avec sa mère en sortant de la DASS, mais cette expérience a échoué. Elle a rencontré un sans domicile avec lequel elle a eu un enfant, confié à une institution de placement. Elle a subi des violences sexuelles de la part du père de son enfant, dont elle est séparée, qui menait une double vie. La manière dont Aude se définit, comme une "sauteuse d'obstacles ", se retrouve tout à fait dans sa vie affective et sexuelle. Face à ce qu'elle considère comme une épreuve, elle se montre combative. Par exemple, son activité sexuelle n'est pas limitée du fait de contraintes écologiques. Aude a débuté sa sexualité alors qu'elle était dans la rue, et n'a pas exprimé de problème particulier pour l'exercer. Elle allait à l'hôtel ou dans le parking de Montparnasse dont elle connaissait le gardien, comme lors de son dernier rapport sexuel, peu de temps avant l'entretien :

"Ça s'est passé où [dernier rapport sexuel] ? / Ben au parking. / On peut avoir des relations sans qu'il y ait d'autres gens autour? / C'est des portes closes. /C'est des petits boxes ? / Oui, l'agent de sécurité nous laisse tranquilles parce qu'il sait qu'on est à la rue, donc il nous protège, enfin c'est un genre de protection./II sait que vous êtes là ? / Ouais, que personne doit rentrer là. Quand y a des gens quidescendent pour prendre leur voiture, il leur dit : non, vous prenez l'ascenseur. II garde... c'est un moyen de sécurité. "

Concernant la protection des rapports sexuels, Aude, bien qu'ayant eu trois interruptions volontaires de grossesse avant la naissance de son fils, déclare "toujours se protéger ". Elle serait phobique de toute maladie, et agit en conséquence : " Vous vous êtes protégée [lors du dernier rapport sexuel] ? / Moi je me protège toujours, j'ai trop peur d'attraper quelque chose. Je vous dis, c'est dans ma tête, j'ai toujours peur d'attraper quelque chose. C'est pour ça, je me protège et après je me lave, au moins 15 minutes en dessous la douche. "Aude a également un recours aux soins qui, s'il n'est pas " toujours" préventif, est au moins curatif: elle consulte au moindre signe, boutons sur la peau, saignement en dehors des règles. Cependant, elle a décidé d'aller se faire poser un implant contraceptif car elle oublie souvent sa pilule, ce que l'on peut qualifier d'attitude préventive. Comme face à toute chose qu'elle considère comme un problème, Aude s'efforce de le résoudre rapidement.

De fait, vivre dans la rue n'est même pas forcément un obstacle, et peut se révéler une opportunité pour mener à bien certains projets. Par exemple, des femmes qui s'éloignent volontairement de leur mari présentent l'hébergement d'urgence comme un refuge et comme un lieu de ressourcement, affectif notamment. S'y affirme la volonté de rupture conjugale, s'y dessinent de nouvelles attentes affectives, s'y rencontrent des partenaires qui confirment la rupture et les nouvelles directions que l'on s'est données. Pour ces femmes, la sexualité est un domaine valorisé et son exercice est valorisant. La faillite conjugale est conséquemment celle de relation sexuelle. Vivre après la séparation implique, de fait, une redéfinition du rapport, auparavant primordial, à la sexualité. L'attention se déplace davantage vers les enfants, et vers soi, notamment pour continuer à plaire. La désunion n'implique pas non plus un moindre souci de soi. En témoigne une grande attention portée à son corps et à son hygiène, qui marque d'ailleurs une différence importante avec d'autres hébergés, prenant moins soin d'eux.

Pour toutes ces femmes, la signification du recours au soin ne paraît guère affectée par l'existence sans domicile ; la perception de risques accrus augmente par contre la fréquence du recours. Ne s'observe pas chez ces femmes, et à la différence des fatalistes ou résignées, une modification sensible du rapport au corps, aux soins et aux risques imputable aux conditions de vie, ou plus exactement au renoncement progressif à faire face aux exigences supplémentaires imposées au maintien de soi par un environnement extrêmement contraignant. La rencontre amoureuse n'est pas envisagée non plus, comme chez les résignées ou les volontaires, comme un médium possible pour sortir de la rue, toutes ces personnes mobilisant d'autres ressources pour se dépêtrer. Que la sexualité soit suspendue ou maintenue, la signification 
que confèrent ces femmes à leur vie affective n'a pas changé avec leur arrivée dans la rue, bien que leurs pratiques aient pu être modulées en fonction des obstacles rencontrés dans l'exercice de l'intimité, ou des priorités qu'elles s'assignent (énoncées sans référence au stigmate de SDF).

\section{Conclusion}

Les expériences affectives et sexuelles des femmes sans-abri apparaissent diversifiées. Leurs discours contredisent de toute évidence le déni, les clichés et le misérabilisme qui caractérisent la plupart des descriptions de leur vie affective et sexuelle. Si les conditions de vie difficiles tendent à contribuer, chez les femmes dont l'univers de sens est la rue, à un délaissement de soi, à l'adoption de ce que nous appellerions des conduites à risques, à une exposition accrue à des agressions, sexuelles notamment, à une réduction de la vie affective et sexuelle à une sphère de personnes proches et tout aussi fatalistes, elles ne sauraient en aucun cas expliquer la diversité de l'expérience des femmes interrogées. Non seulement des femmes qui se considèrent sans-abri exercent une sexualité désirée, et connectent leur désir à leur volonté de sortir de la rue, mais des femmes qui vivent sans domicile sans que la rue ne constitue leur univers de sens se montrent également débrouillardes dans un environnement physique et relationnel diversement contraignant. Leurs expériences s'enracinent en effet dans des univers de sens qui rendent la variable écologique peu pertinente, nous semble-t-il, pour rendre compte de la signification de leur vie affective et sexuelle, comme de leurs conduites face aux risques.

Demandons-nous enfin si cette présentation de la vie affective et sexuelle de femmes sans-abri concerne spécifiquement des femmes. En effet, l'enquête dont sont tirés ces entretiens cherchait à comprendre l'expérience affective et sexuelle de personnes sansabri, mais pas de femmes en particulier. En outre, la classification des entretiens ne distinguait pas, a priori, hommes et femmes, si bien que des femmes dont nous avons parlé côtoient des hommes dans les groupes que nous avons construits. De fait, si nous avons bel et bien décrit la vie affective et sexuelle de femmes dans la rue, les univers de sens dans lesquels s'inscrivent leurs expériences ne sont pas clivés selon le genre : des hommes comme des femmes se montrent fatalistes, résignés ou volontaires, par rapport au stigmate qui les affligent, entre autres dans leurs relations affectives; des hommes comme des femmes sont en rupture momentanée avec l'insertion et subordonnent leur vie affective et sexuelle à une stabilisation professionnelle et résidentielle, et ainsi de suite. En revanche, les modalités que prennent, dans la sphère affective et sexuelle notamment, des conduites fatalistes, résignées, ou volontaires, sont en partie liées au genre : ainsi le délaissement de soi des femmes se manifeste-t-il par une perte reconnue de féminité ; de même, la volonté de sortir de la rue peut se traduire par des jeux de séduction mettant à profit la féminité (tandis que des hommes en pareille situation se refuseront à s'engager dans des relations, même légères, n'ayant pas les moyens pécuniaires d'honorer leur partenaire). Semblablement, pour des personnes dont l'univers de sens n'est pas la rue, la manière de répondre aux questions par lesquelles elles définissent (indifféremment au sexe) leur présence en centre d'hébergement, et qui orientent leurs expériences affectives et sexuelles, ne parait pas dépendre systématiquement du genre. Le genre, en demeurant un " discriminant de rôle " [17], ne saurait donc suffire à rendre compte ni des problèmes ni des solutions pratiques mises en œuvre par les sans-abri.

\section{Références bibliographiques}

1. Pichon P. Premiers travaux sociologiques et ethnographiques français à propos de sans domicile fixe. In La rue et le foyer, M Marpsat et JM Firdion, Eds. Paris : PUF/Ined, 2000, p. 107-123.

2. Edgar B et Doherty J. Women and Homelessness in Europe. Bristol : The Policy Press, 2001.

3. Marpsat M. Un avantage sous contrainte. Le risque moindre pour les femmes de se trouver sans abri. Population $1999 ; 54$ (6) : 885-932.

4. Lanzarini C. Survivre dans le monde sous-prolétaire. Paris : PUF, 2000.

5. Declerck P. Les naufragés. Avec les clochards de Paris. Paris : Plon, 2001.

6. Bruneteaux P. Lanzarini C. La sexualité agressée des sous-prolétaires à la rue. L'Homme et la société $1998 ; 129: 113-126$.

7. Brunet L, Carpentier S, Laporte A, Pourette D et al. Féminité, Accès aux soins, Maternité et Risques vécus par les femmes en grande précarité. Une contribution à l'amélioration de leur santé gynécologique. Rapport à la Direction générale de la santé, Paris : Observatoire du Samusocial de Paris, 2005.

8. Laporte A, Le Méner E, Pourette D. Oppenchaim N et al., Survivre ou faire l'amour. La pluralité des expériences affectives et sexuelles de personnes sans - domicile fixe. Rapport à l'Institut national de prévention et d'éducation à la santé. Paris : Observatoire du Samusocial de Paris, 2007.

9. Thomas WI. Définir la situation. In L'École de Chicago. Naissance de l'écologie urbaine, Y Grafmeyer et I Joseph, Eds. Paris : Flammarion, 2004, p. 79-82.

10. Weber M. Économie et société, tome 1 : Les catégories de la sociologie. Paris : Plon, 1995, p. 48-52.

11. Beaud S. L'usage de l'entretien en sciences sociales. Plaidoyer pour l'a entretien ethnographique $*$. Politix $1996 ; 35: 226-257$.

12. Goffman E. Stigmate. Les usages sociaux des handicaps. Paris : Editions de Minuit, 1975.

13. Gaboriau P. Clochard. L'univers d'un groupe de sans-abri parisien, Paris, Julliard, 1993.

14. Paugam S. La disqualification sociale. Paris : PUF, 1991

15. Goffman E. La mise en scène de la vie quotidienne. Les relations en public. Paris : Editions de Minuit, 1973.

16. Paugam S. L'exclusion. L'état des savoirs. Paris : PUF, 1996.

17. Hannerz U. Explorer la ville. Paris : Editions de Minuit, 1980. 